\title{
Enhancement of GLI1-transcriptional activity by B-catenin in human cancer cells
}

\author{
OSAMU MAEDA ${ }^{1}$, MASASHI KONDO ${ }^{2}$, TAKAYOSHI FUJITA ${ }^{1}$, NORIYASU USAMI ${ }^{3}$, TAKAYUKI FUKUI ${ }^{3,4}$, \\ KAORU SHIMOKATA ${ }^{2}$, TAKAFUMI ANDO ${ }^{1}$, HIDEMI GOTO ${ }^{1}$ and YOSHITAKA SEKIDO $^{4}$ \\ Departments of ${ }^{1}$ Gastroenterology, ${ }^{2}$ Respiratory Medicine, and ${ }^{3}$ Thoracic Surgery, Nagoya University Graduate School of \\ Medicine, Tsurumai-cho 65, Showa-ku, Nagoya 466-8550; ${ }^{4}$ Division of Molecular Oncology, Aichi Cancer \\ Center Research Institute, Kanokoden 1-1, Chikusa-ku, Nagoya 464-8681, Japan
}

Received January 24, 2006; Accepted March 13, 2006

\begin{abstract}
The Hedgehog (Hh) signaling pathway and the Wnt signaling pathway are known to play important roles in carcinogenesis and the progression of various human malignant tumors. Although a relationship between these two pathways has recently been reported, the mechanism by which B-catenin, one of the key molecules of the Wnt signaling pathway, influences the Hh pathway has not yet been revealed in detail. To clarify the role of $B$-catenin in relation to the Hh signaling pathway, we transfected GLI1 and $\beta$-catenin expression constructs into human malignant cells, including stomach, colon, and lung cancers, and evaluated the luciferase activity of GLI-responsive reporter constructs. While exogenous GLI1 increased the luciferase activity, exogenous B-catenin also enhanced the activity under overexpression of GLI1. However, co-transfection with T-cell factor (TCF)-4 or lymphocyte enhancer factor (LEF)-1 did not influence the activity, indicating that the enhancement of $\beta$-catenin in relation to the $\mathrm{Hh}$ signaling pathway is not TCF/LEFdependent. Our results suggest that $\beta$-catenin might be involved in the Hh signaling pathway via enhancement of the transcriptional activity of GLI.
\end{abstract}

\section{Introduction}

The Hedgehog (Hh) signaling pathway is critical for growth and patterning during embryonic development (1), and its disregulation has been reported to lead to various human diseases including birth defects and cancers (2). Molecules involved in this pathway, which transmits signals from extracellular spaces to the nucleus, have been well characterized

Correspondence to: Dr Yoshitaka Sekido, Division of Molecular Oncology, Aichi Cancer Center Research Institute, Kanokoden 1-1, Chikusa-ku, Nagoya 464-8681, Japan

E-mail: ysekido@aichi-cc.jp

Key words: ß-catenin, GLI1, Wnt, Hedgehog in detail. Briefly, Hh ligands bind to a transmembrane receptor protein, patched (Ptch), which, in the absence of $\mathrm{Hh}$, exerts an inhibitory effect on the seven transmembrane receptor smoothened (Smo). Binding of Hh ligands to Ptch blocks the inhibitory effect of Ptch on Smo. Activated Smo induces a complex series of intracellular reactions that targets the Cubitus Interruptus (Ci)/Gli family of zinc finger transcription factors (3). In vertebrates, three Gli genes are present, with Gli1 being predominantly a transcriptional activator while Gli2 and Gli3 act as both activator and repressor (2).

Some human malignancies, including basal cell carcinoma, medulloblastoma, and rhabdomyosarcoma, have been shown to be associated with mutations which activate Smo or with those which inactivate Ptch. Recently, it has also been reported that Hh ligand stimulation is essential for the growth of gastrointestinal cancers without a mutation in Smo or Ptch (4). The Hh signaling pathway has also been suggested to be involved in the development of other human malignancies, including pancreatic $(5,6)$, lung $(7)$, breast $(8)$, prostate $(9)$, and colorectal cancers (10).

Meanwhile, the Wnt signaling pathway is known to be involved in a wide range of embryonic patterning events, and its disregulation plays a critical role in the development of various kinds of human cancers $(11,12)$. B-catenin is one of the key molecules in this signaling pathway, which also participates in cell adhesion with cadherins. In the presence of Wnt signals, a constitutively activating mutation of B-catenin, or an inactivating mutation of adenomatous polyposis coli (APC), the cytoplasmic pool of $\beta$-catenin increases and accumulated $B$ catenin enters the nucleus. This results in the formation of complexes with T-cell factor (TCF)/lymphocyte enhancer factor (LEF) family proteins, which are capable of transactivating their target genes that encode proteins involved in cell growth and proliferation such as cyclin D.

Although recent studies suggest that a relationship between the Hh pathway and the Wnt pathway may exist (13), the role of $\beta$-catenin in the Hh signaling pathway has not been revealed in detail. To clarify the role of $B$-catenin in the Hh signaling pathway, we investigated the function of $\beta$-catenin using luciferase reporter assays under co-transfection of $\beta$-catenin and GLI 1 constructs. In the present study, we showed that $\beta$ catenin enhances the luciferase activity of the GLI-responsive reporter construct under overexpression of GLI1, but that 
TCF/LEF does not enhance the GLI-responsive reporter activity by $\beta$-catenin. Our results suggest that $\beta$-catenin may potentially affect the GLI1 transcriptional activity via a TCF/LEF independent manner.

\section{Materials and methods}

Plasmids. The GLI1 expression construct, GLI1-V5, was synthesized by cloning a PCR product of the coding region amplified from pSR $\alpha$-GLI1 (14) into pcDNA3.1/V5-HisA (Invitrogen, Carlsbad, CA). The B-catenin, TCF-4 and LEF-1 expression constructs are described elsewhere $(15,16)$. 8x3'Gli-BS Luc and 8xm3'Gli-BS Luc were provided by Dr H. Sasaki. The $8 \times 3$ 'Gli-BS Luc contains eight GLI1 binding sites, and $8 \times \mathrm{xm}^{\prime}$ 'Gli-BS Luc contains mutated inactive binding sites (17). pGL3-OT and pGL3-OF, containing a promoter including three TCF/LEF binding sites and mutated inactive binding sites, respectively, were provided by Dr B. Vogelstein. A wild-type human GLI2 expression construct was provided by Dr E. Roessler (18).

Cell lines. NCI-H1299, a lung cancer cell line, was a gift from Dr A.F. Gazdar. AGS and KATO III, gastric cancer cell lines; NCI-H28 (CRL5820), a malignant mesothelioma cell line; and SW-480, a colon cancer cell line; were purchased from the American Type Tissue Culture Collection (ATCC) (Rockville, MD). HGC-27, a gastric cancer cell line; and CW-2 and COLO-320, colon cancer cell lines; were purchased from RIKEN BioResource Center (Tsukuba, Japan).

Cell culture and transient transfection. All cell lines used except SW-480 were cultured in RPMI-1640 (Invitrogen) supplemented with $10 \%$ fetal bovine serum (Equitech-Bio, Ingram, TX), and 1X Antibiotic-Antimycotic (Invitrogen) at $37^{\circ} \mathrm{C}$ in a humidified incubator containing $5 \% \mathrm{CO}_{2} . \mathrm{SW}-480$ was cultured in Leibovitz L15 (Invitrogen) supplemented with $10 \%$ fetal bovine serum and $1 \mathrm{X}$ Antibiotic-Antimucotic at $37^{\circ} \mathrm{C}$ in a humidified incubator without $\mathrm{CO}_{2}$. Transient transfection for cells except KATO III was performed using Lipofectamine Plus reagent (Invitrogen) according to the manufacturer's protocols, and Lipofectamine 2000 (Invitrogen) was used for KATO III.

RT-PCR analysis. Total RNA was extracted from cell lines using TRIzol reagent (Invitrogen). First-strand cDNA was generated using SuperScript II (Invitrogen) and random primers (Takara Bio, Otsu, Japan) according to the manufacturer's instructions. RT-PCR was performed using the modified touchdown PCR method (19). The sequences of primers used in this study were as follows: 5'-TCTGCTGCTAGTCCTC GTCT-3' (forward) and 5'-TAGTACACCCAGTCGAA GCC-3' (reverse) for Sonic Hedgehog (SHH), 5'-GTCAT CCCCTTTGTCCTCA-3' (forward) and 5'-GATCTCACA GTCAGGGATGG-3' (reverse) for Smoothened (SMO), 5'GTGGAAGTTGGAGGACGAGT-3' (forward) and 5'CACAATCAACTCCTCCTGCC-3' (reverse) for Patched (PTCH), 5'-CAAGAATTCAGCACATGCTG-3' (forward) and 5'-GGAAGGACAGGTTTGCTGTT-3' (reverse) for SUFU, and 5'-TCCCATCACCATCTTCCAG-3' (forward) and 5'-ATGAGTCCTTCCACGATACC-3' (reverse) for GAPDH.
Western blot analysis. Preparation of total cell lysates and Western blotting were performed as described previously (20). The first antibodies used were anti-ß-catenin antibody (C19220, Pharmingen/Transduction Laboratories, San Diego, CA), anti-V5 antibody (R960-25, Invitrogen), anti-GLI1 antibody (sc-20687, Santa Cruz Biotechnology, CA), anti-c-myc antibody (SA-294, BIOMOL, PA), and anti-actin antibody (A-2066, Sigma, MO).

Immunofluorescence staining. Cells were grown on cover glasses in RPMI-1640 media for $24 \mathrm{~h}$ at $37^{\circ} \mathrm{C}$. After three washes with phosphate-buffered saline (PBS), cells were fixed in $4 \%$ paraformaldehyde/PBS for $15 \mathrm{~min}$, permeabilized with $0.2 \%$ Triton $\mathrm{X}-100 / \mathrm{PBS}$ for $5 \mathrm{~min}$, and pre-blocked in $5 \%$ nonfat dry milk/PBS for $15 \mathrm{~min}$. The slides were then incubated with a primary antibody (diluted 1:500 in blocking solution) for $1 \mathrm{~h}$, washed three times with PBS, and incubated with a secondary antibody with a FITC-conjugated anti-mouse antibody (FI-2000, Vector Laboratories, CA) for $30 \mathrm{~min}$. After further washes, the slides were mounted with Vectashield (Vector Laboratories), and cells were examined with fluorescence optics on a confocal microscope and photographed at a x600 magnification.

Luciferase reporter assay. Cells grown in 24-well tissueculture plates were transfected with $100 \mathrm{ng}$ of one of the ß-catenin constructs, $10 \mathrm{ng}$ of the GLI1, TCF- 4 or LEF-1 constructs, $100 \mathrm{ng}$ of $8 \times 3$ 'Gli-BS Luc or $8 \times \mathrm{xm} 3$ 'Gli-BS Luc, pGL3-OT or pGL3-OF, and $2 \mathrm{ng}$ of phRL-TK vector (Promega). Forty-eight hours after transfection, reporter gene activities were determined in a luminometer with the DualLuciferase reporter assay system (Promega).

\section{Results}

$\beta$-catenin enhances the luciferase activity of the GLIresponsive reporter. To determine whether $\beta$-catenin has an ability to enhance the transcriptional activity of GLI1, we transfected NCI-H1299 cells with one of the B-catenin expression constructs or an empty vector together with GLIresponsive reporter, $8 \times 3{ }^{\prime}$ Gli-BS Luc or $8 \times m 3$ 'Gli-BS Luc. First, we found that the luciferase activity of the GLIresponsive reporter construct was enhanced with the GLI1 expression construct, but not changed when transfected with only wild-type or an activated type of B-catenin; the latter lacks a serine residue at the GSK3ß phosphorylation site (S37C) (Fig. 1A). Next, we co-transfected the GLI1 expression construct with wild-type or an activated type of $\beta$-catenin, and found that the activity was enhanced more strongly, and that the amount of enhancement caused by $\beta$-catS37C was larger than that of wild-type $\beta$-catenin (Fig. 1A).

To test the possibility that the enhancement of the transcriptional activity of GLI1 is because exogenous B-catenin increases the amount of expression and/or changes the intracellular distribution of exogenous GLI1, we performed Western blot analysis and immunofluorescence staining. When NCI-H1299 cells were transfected with GLI1-V5 with or without one of the $\beta$-catenin expression constructs, we did not detect any difference in the expression levels of GLI1 (Fig. 1B). Immunofluorescence staining also demonstrated that the 
A

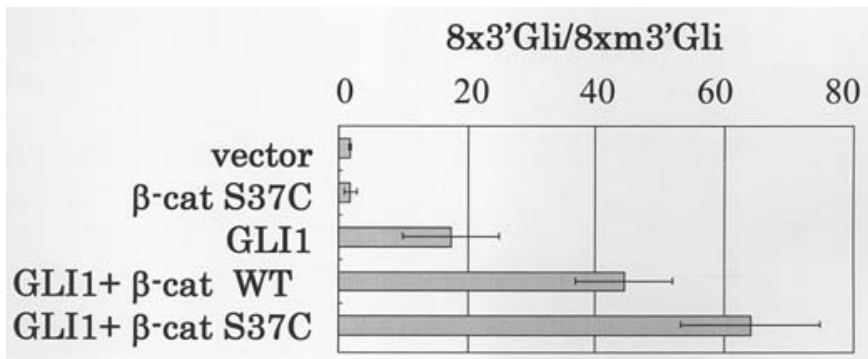

B

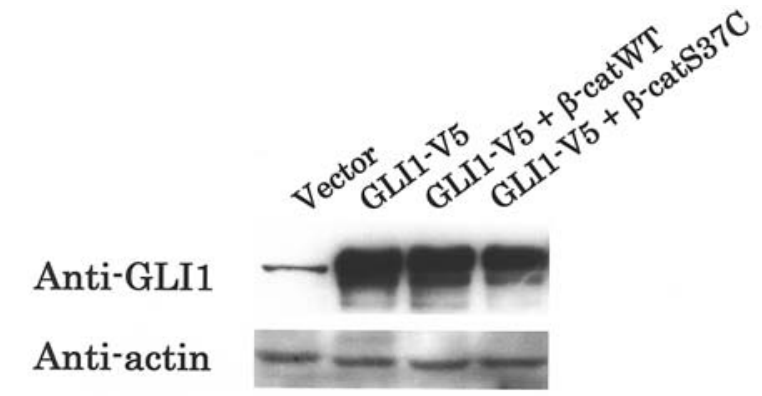

Anti-V5

Anti-actin
$\mathrm{C}$

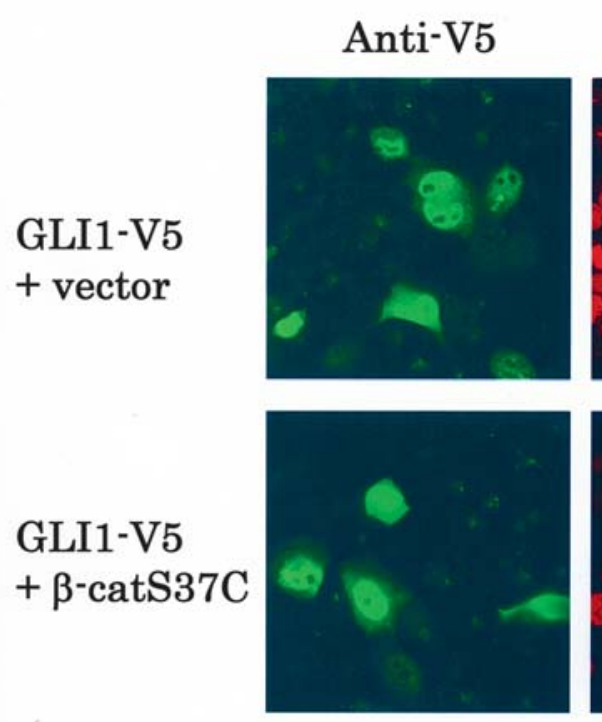

$\mathrm{D}$

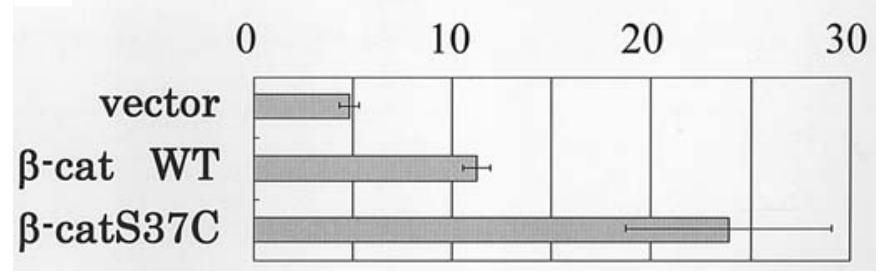

Figure 1. B-catenin enhances GLI-responsive reporter activity. (A) Luciferase reporter assay using GLI-responsive reporter. NCI-H1299 cells were transfected with $8 \times 3$ 'Gli-BS Luc or $8 \times 3 \mathrm{~m}^{\prime}$ Gli-BS Luc together with $B$ catenin expression construct and/or GLI1 expression construct. The ratios of normalized luciferase activities between $8 \times 3{ }^{\prime}$ Gli-BS Luc and 8xm3'Gli-BS Luc were determined. (B) Expression of GLI1 with or without B-catenin cotransfection. After NCI-H1299 cells were transfected with the GLI1-V5 expression construct and each of two B-catenin expression constructs, Western blot analysis was performed with anti-GLI1 antibody and anti-V5 antibody; the former detects both endogenous and exogenous GLI1 while the latter detects only exogenous GLI1. (C) Immunofluorescence staining of GLI1-V5. NCI-H1299 cells were transfected GLI1-V5 with or without activated B-catenin. Exogenous GLI1-V5 was found in the nucleus of the transfected cells. PI, propidium iodide. (D) Luciferase reporter assay using GLI1 stably-transfected cells. NCI-H1299 cells were transfected with GLI1V5 and selected with G418 for two weeks. The established cells stably expressing GLI1were transiently transfected with one of the $\beta$-catenin constructs and reporter constructs, which were then tested by luciferase assay.

intracellular distribution of GLI1, which was predominant at the nuclei, was not changed with or without co-transfection of ß-catenin (Fig. 1C). Furthermore, we also established NCI-
H1299 cells with a stably abundant expression of GLI1-V5 by G418 selection in order to test the enhancement of luciferase activity by $\beta$-catenin at a more constant background level of GLI1 expression. When these cells were transfected with the ß-catenin expression construct, we confirmed that exogenous B-catenin similarly enhanced the luciferase activity of the GLI-responsive reporter (Fig. 1D). Thus, these results suggest that $\beta$-catenin itself might have the ability to enhance the transcriptional activity of GLI1, not due to an increase or intracellular translocation of GLI1.

TCF-4 or LEF-1 does not increase the luciferase activity of the GLI-responsive reporter. $B$-catenin transcriptional activation for most of its target genes is thought to be dependent on the TCF/LEF transcription factor. To study whether the effect of $B$-catenin on the GLI-responsive reporter is also induced through TCF/LEF, we co-transfected TCF-4 or LEF-1 expression constructs into NCI-H1299 cells (Fig. 2). First, we confirmed that exogenous B-catenin enhances the TCF/LEF-dependent transcriptional activity using pGL3-OT and pGL3-OF luciferase reporters, which were further increased by TCF-4 or LEF-1 co-transfection. However, GLI1 transfection did not affect the transcriptional activity of the TCF/LEF-dependent reporter, regardless of B-catenin co-transfection (Fig. 2A). Next, we transfected 
A

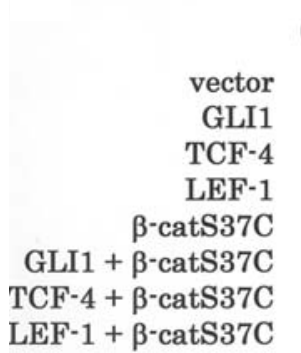

$\mathrm{C}$

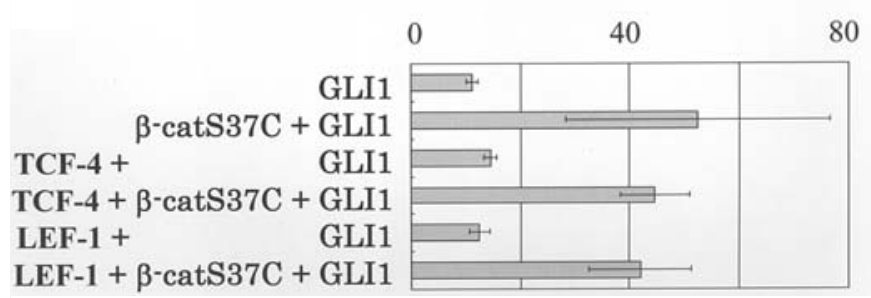

TCF/LEF with or without $\beta$-catenin, and tested GLIresponsive reporter activity. We found that overexpression of TCF-4 or LEF-1 did not increase the luciferase activity of the GLI-responsive reporter (Fig. 2B). Finally, we co-transfected NCI-H1299 cells with GLI1 and TCF/LEF, but did not find that TCF/LEF increases the luciferase activity of the GLIresponsive reporter (Fig. 2C). Thus, these results suggest that the effect of $B$-catenin on the Hh signaling pathway is independent of TCF/LEF activity.

$\beta$-catenin enhances GLI-responsive reporter activity in various cell lines. To test whether $\beta$-catenin also enhances GLI transcriptional activity in other human malignant tumor cells, we studied another seven cancer cell lines. First, we performed reverse transcriptase (RT)-PCR to analyze the expression levels of components in the Hh pathway, $\mathrm{SHH}$, PTCH, SMO and SUFU, and Western blot analysis for GLI1 and B-catenin (Fig. 3A). Although there were differences in the expression levels, expression of PTCH and SUFU was detected in all cell lines tested. Meanwhile, the expression levels were quite distinct for $\mathrm{SHH}$ and $\mathrm{SMO}$, with some being strong expressors and the others null expressors. GLI1 and $\beta$-catenin expression was detected in all cell lines except $\beta$-catenin in NCI-H28, a $\beta$-catenin-deficient malignant mesothelioma cell line (21). Next, with the luciferase reporter assay, we found that exogenous B-catenin enhanced the luciferase activity in all three gastric cancer cell lines and two of three colon cancer cell lines when GLI1 was overexpressed (Fig. 3B). The luciferase activity of GLI1 was also enhanced in NCI-H28 cells with exogenous B-catenin (Fig. 3C), suggesting that endogenous $\beta$-catenin is not essential for GLI1 to activate the reporter activity.

$\beta$-catenin enhances the luciferase activity of the GLI-responsive reporter induced by GLI2. To test whether B-catenin enhances the activity of other GLI transcriptional factors in the $\mathrm{Hh}$ signaling pathway, we transfected H1299 cells with GLI2 with or without the $B$-catenin construct. The luciferase activity of the GLI-responsive reporter by GLI2 was shown
B

80

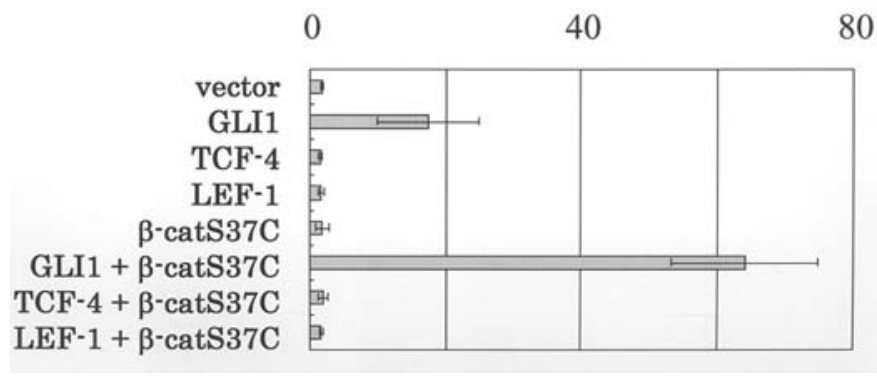

Figure 2. TCF-4 or LEF-1 does not change the luciferase activity of the GLIresponsive reporter. (A) Luciferase assay using TCF/LEF-responsive reporter, pGL3-OT and pGL3-OF. Luciferase activity of B-catenin was strongly enhanced by TCF-4 and LEF-1, but not by GLI1. (B) Luciferase assay using GLI-responsive reporter. Luciferase activity of GLI1 was enhanced by Bcatenin but not by TCF-4 or LEF-1. (C) Luciferase assay using GLI-responsive reporter under co-transfection with GLI1. TCF-4 or LEF-1 did not increase the activity under overexpression of GLI1.

to be enhanced by co-transfection with the $\beta$-catenin construct (Fig. 4A). Since Western blot analysis revealed that co-transfection with B-catenin did not change the expression level of GLI2, the enhancement of GLI2 transcriptional activity was suspected to be independent of an increase in the amount of GLI2 by B-catenin (Fig. 4B).

\section{Discussion}

In the present study, we demonstrated that $\beta$-catenin enhances the activity of the GLI-responsive reporter under co-transfection with GLI1 using GLI-responsive reporter assay. Since TCF/LEF co-transfection did not change the activity, the enhancement of $B$-catenin in relation to the Hh signaling pathway was suggested to be TCF/LEF-independent.

Gli family genes are downstream transcriptional factors, of which GLI1 itself is also thought to be one of the transcriptional targets of the Hh signaling pathway, and one of the major mechanisms to control the function of Cubitus Interruptus (Ci), the homologue of Gli in Drosophila, is processing into a Cterminal truncated repressor form (22). In vertebrates, Gli2 and Gli3 have been shown to undergo proteolytic cleavage $(23,24)$, but Gli1 does not appear to undergo the cleavage nor function as a repressor (25). Although the expression level and distribution in the nucleus of Gli1 seem to be important to control the activity, the mechanisms for controlling Gli transcriptional output remain poorly understood (1).

Disturbance in the Hh signaling pathway has been reported to be involved in carcinogenesis and tumor progression in various human malignancies. GLI1 was reported to be amplified in some osteosarcomas, rhabdomyosarcomas, and B cell lymphomas (22). Overexpression of GLI1 was shown in basal cell carcinomas of the skin (26) and in bone and softtissue sarcomas, with the expression degree in the latter correlating with the tumor grade (27). Furthermore, mutations of PTCH and SMO were also detected in basal cell carcinomas and in medulloblastomas.

Several reports previously indicated a relationship between the Wnt signaling pathway and the Hh signaling pathway, 
A
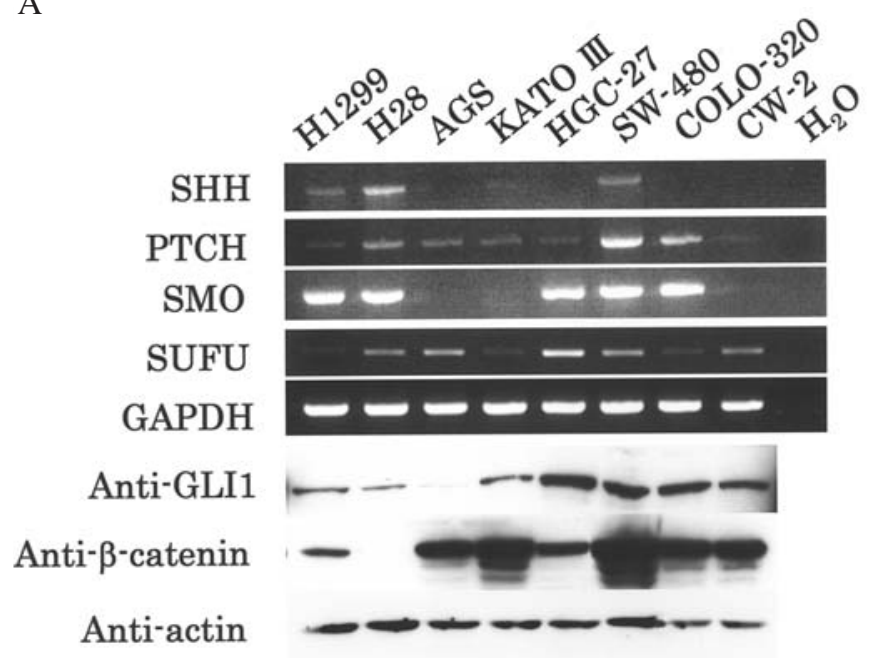

$\mathrm{B}$

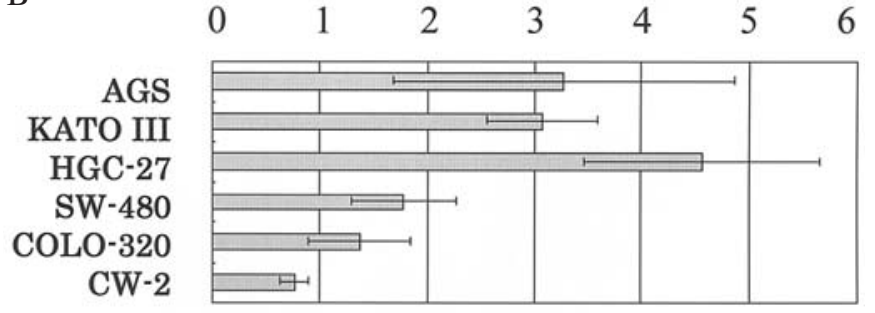

C

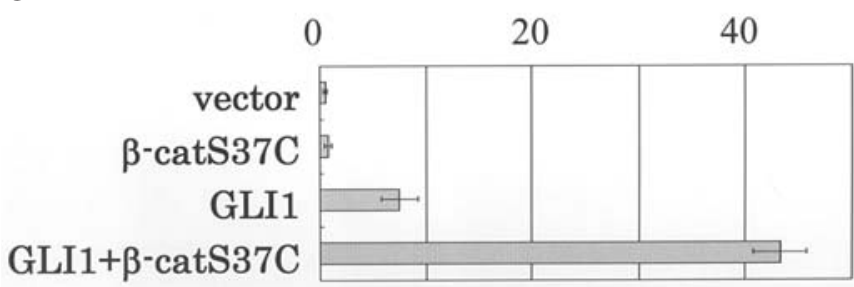

Figure 3. Expression analysis and luciferase reporter assay using GLI-responsive reporter in various types of human cancer cells. (A) RT-PCR analysis of SHH, PTCH, SMO and SUFU, and Western blot analysis of GLI1 and B-catenin. (B) Luciferase reporter assay using gastric cancer cell lines AGS, KATO III, and HGC-27, and colon cancer cell lines SW-480, COLO-320, and CW-2. The ratios of luciferase activity of cells co-transfected with both GLI1 and B-catenin to those with only GLI1 are shown. (C) Luciferase reporter assay using a ß-catenin-deficient malignant mesothelioma cell line, NCI-H28.

A

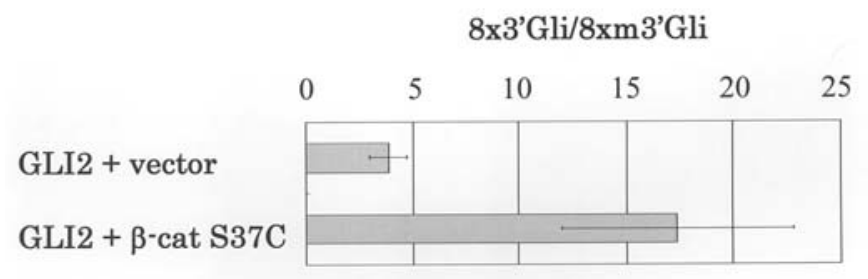

B

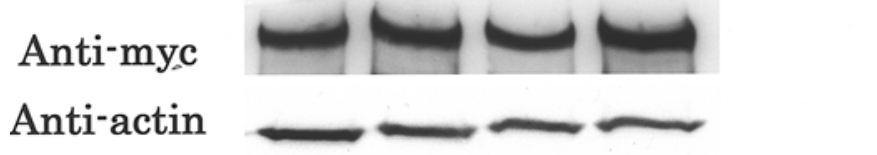

Figure 4. B-catenin enhance reporter activity induced by GLI2. (A) Luciferase reporter assay using H1299 cells. (B) H1299 cells were transfected with GLI2 expression construct with or without B-catenin, and GLI2 expression was detected with anti-myc antibody. Transfections were done in duplicate.

suggesting the existence of cross-talk between these two signaling cascades. For example, the Wnt family has been shown to be one of the target genes of the Hh signaling pathway (23), and a suppressor of fused (SUFU), which interacts with GLI and negatively regulates $\mathrm{Hh}$ signaling, and was also reported to inhibit both pathways (13). Furthermore, in the differentiation of colon epithelium, Indian Hedgehog and Wnt signaling were also reported to suppress each other (28). This is intriguing, since it suggests that the abnormality of one of these two pathways may affect the other, which may together contribute to the development of colon cancer. However, how these two signaling pathways interact with each other remains unclear.

We showed that exogenous $\beta$-catenin enhanced the luciferase activity of the GLI-responsive reporter under overexpression of GLI1, suggesting that $\beta$-catenin may increase the activity of the $\mathrm{Hh}$ signaling pathway. Since cotransfection of TCF-4 or LEF-1 did not increase the activity, we considered that the enhancement is not due to TCF/LEFdependent transcriptional activity. To test whether $\beta$-catenin and GLI1 form a complex, we also performed immunoprecipitation assay using cells which were overexpressed with exogenous B-catenin and GLI1, but did not detect complex formation (data not shown). Thus, although the mechanism is yet unclear, we suspect that $\beta$-catenin enhances GLI transcriptional activity but probably not due to direct contact.

We also analyzed whether transfection of $\beta$-catenin alone, without GLI1, also enhances the GLI-responsive reporter activity. Under the expression level of endogenous GLI1, exogenous $\beta$-catenin did not induce enhancement of GLIresponsive reporter activity in various types of cells. Instead, an excess amount of exogenous GLI1 was always shown to be necessary to detect a sufficient effect of B-catenin for GLItranscriptional activity. The reason why endogenous GLI1 was insufficient for B-catenin to act as a coactivator of GLI1 remains unclear. Lastly, we also tried to determine whether the change in endogenous $\beta$-catenin around its basal level also affects the activity of the Hh pathway. To test this, we used siRNA for B-catenin to suppress the endogenous B-catenin. We found that transfection of $\beta$-catenin siRNA slightly reduced the luciferase activity of the GLI-responsive reporter in HGC-27 cells but not in other cancer cells (data not shown). 
Thus, we speculate that endogenous B-catenin may also function on the Hh pathway through an unknown mechanism indirectly in a specific type of cancer cells.

In the present study, we demonstrated that $\beta$-catenin may potentially enhance the transcriptional activity of Gli in the Hh signaling pathway. In addition to disregulating the Wnt signaling pathway by its activating mutation in various human malignancies, $\beta$-catenin may also play an activation role in the Hh signaling pathway. Thus, $B$-catenin activation seems to be involved in tumorigenesis with multiple aspects, which may further strengthen the rationale that $B$-catenin can be one of the optimum molecules for molecular-targeted therapy.

\section{Acknowledgements}

We thank Dr Bert Vogelstein for providing pGL3-OT and pGL3-OF, Dr Hiroshi Sasaki for providing 8x3'Gli-BS Luc and $8 \times m 3$ 'Gli-BS Luc, Dr Shunsuke Ishii for the pSR $\alpha-G L I 1$ and pSR $\alpha$-GLI3, Dr Adi F. Gazdar for the NCI-H1299 cell line, Dr Eric Roessler for the GLI2 construct, and Ms. Hiroko Kaga and Ms. Chie Moriyama for technical support.

\section{References}

1. Ingham PW and McMahon AP: Hedgehog signaling in animal development: paradigms and principles. Genes Dev 15: 3059-3087, 2001.

2. Ruiz i Altaba A, Sanchez P and Dahmane N: Gli and hedgehog in cancer: tumours, embryos and stem cells. Nat Rev Cancer 2: 361-372, 2002.

3. Methot $\mathrm{N}$ and Basler $\mathrm{K}$ : An absolute requirement for Cubitus interruptus in Hedgehog signaling. Development 128: 733-742, 2001.

4. Berman DM, Karhadkar SS, Maitra A, Montes De Oca R, Gerstenblith MR, Briggs K, Parker AR, Shimada Y, Eshleman JR, Watkins DN and Beachy PA: Widespread requirement for Hedgehog ligand stimulation in growth of digestive tract tumours. Nature 425: 846-851, 2003.

5. Thayer SP, di Magliano MP, Heiser PW, Nielsen CM, Roberts DJ, Lauwers GY, Qi YP, Gysin S, Fernandez-del Castillo C, Yajnik V, Antoniu B, McMahon M, Warshaw AL and Hebrok M: Hedgehog is an early and late mediator of pancreatic cancer tumorigenesis. Nature 425: 851-856, 2003.

6. Kayed H, Kleeff J, Keleg S, Guo J, Ketterer K, Berberat PO, Giese N, Esposito I, Giese T, Buchler MW and Friess H: Indian hedgehog signaling pathway: expression and regulation in pancreatic cancer. Int J Cancer 110: 668-676, 2004.

7. Watkins DN, Berman DM, Burkholder SG, Wang B, Beachy PA and Baylin SB: Hedgehog signalling within airway epithelial progenitors and in small-cell lung cancer. Nature 422: 313-317, 2003.

8. Kubo M, Nakamura M, Tasaki A, Yamanaka N, Nakashima H, Nomura M, Kuroki S and Katano M: Hedgehog signaling pathway is a new therapeutic target for patients with breast cancer. Cancer Res 64: 6071-6074, 2004.

9. Sanchez P, Hernandez AM, Stecca B, Kahler AJ, DeGueme AM, Barrett A, Beyna M, Datta MW, Datta S and Ruiz i Altaba A: Inhibition of prostate cancer proliferation by interference with SONIC HEDGEHOG-GLI1 signaling. Proc Natl Acad Sci USA 101: 12561-12566, 2004.

10. Qualtrough D, Buda A, Gaffield W, Williams AC and Paraskeva C: Hedgehog signalling in colorectal tumour cells: induction of apoptosis with cyclopamine treatment. Int J Cancer 110: 831-837, 2004.
11. Peifer M and Polakis P: Wnt signaling in oncogenesis and embryogenesis - a look outside the nucleus. Science 287: 1606-1609, 2000 .

12. Moon RT, Kohn AD, De Ferrari GV and Kaykas A: WNT and B-catenin signalling: diseases and therapies. Nat Rev Genet 5: 691-701, 2004.

13. Taylor MD, Zhang X, Liu L, Hui CC, Mainprize TG, Scherer SW, Wainwright B, Hogg D and Rutka JT: Failure of a medulloblastoma-derived mutant of SUFU to suppress WNT signaling. Oncogene 23: 4577-4583, 2004.

14. Dai P, Akimaru H, Tanaka Y, Maekawa T, Nakafuku M and Ishii S: Sonic Hedgehog-induced activation of the Gli1 promoter is mediated by GLI3. J Biol Chem 274: 8143-8152, 1999.

15. Usami N, Sekido Y, Maeda O, Yamamoto K, Minna JD, Hasegawa Y, Yoshioka H, Imaizumi M, Ueda Y, Takahashi M and Shimokata $\mathrm{K}$ : $\beta$-catenin inhibits cell growth of a malignant mesothelioma cell line, NCI-H28, with a 3p21.3 homozygous deletion. Oncogene 22: 7923-7930, 2003.

16. Maeda O, Usami N, Kondo M, Takahashi M, Goto H, Shimokata K, Kusugami K and Sekido Y: Plakoglobin ( $\gamma$ catenin) has TCF/LEF family-dependent transcriptional activity in B-catenin-deficient cell line. Oncogene 23: 964-972, 2004.

17. Sasaki H, Hui C, Nakafuku M and Kondoh H: A binding site for Gli proteins is essential for HNF-3beta floor plate enhancer activity in transgenics and can respond to Shh in vitro. Development 124: 1313-1322, 1997.

18. Roessler E, Ermilov AN, Grange DK, Wang A, Grachtchouk M, Dlugosz AA and Muenke M: A previously unidentified aminoterminal domain regulates transcriptional activity of wild-type and disease-associated human GLI2. Hum Mol Genet 14: 2181-2188, 2005.

19. Don RH, Cox PT, Wainwright BJ, Baker K and Mattick JS: 'Touchdown' PCR to circumvent spurious priming during gene amplification. Nucleic Acids Res 19: 4008, 1991.

20. Sekido Y, Sato M, Usami N, Shigemitsu K, Mori S, Maeda O, Yokoi T, Hasegawa Y, Yoshioka H and Shimokata K: Establishment of a large cell lung cancer cell line (Y-ML-1B) producing granulocyte colony-stimulating factor. Cancer Genet Cytogenet 137: 33-42, 2002.

21. Shigemitsu K, Sekido Y, Usami N, Mori S, Sato M, Horio Y, Hasegawa Y, Bader SA, Gazdar AF, Minna JD, Hida T, Yoshioka H, Imaizumi M, Ueda Y, Takahashi M and Shimokata K: Genetic alteration of the B-catenin gene (CTNNB1) in human lung cancer and malignant mesothelioma and identification of a new 3p21.3 homozygous deletion. Oncogene 20: 4249-4257, 2001.

22. Villavicencio EH, Walterhouse DO and Iannaccone PM: The sonic hedgehog-patched-gli pathway in human development and disease. Am J Hum Genet 67: 1047-1054, 2000.

23. Ruiz i Altaba A: Gli proteins encode context-dependent positive and negative functions: implications for development and disease. Development 126: 3205-3216, 1999.

24. Wang B, Fallon JF and Beachy PA: Hedgehog-regulated processing of Gli3 produces an anterior/posterior repressor gradient in the developing vertebrate limb. Cell 100: 423-434, 2000 .

25. Cohen MM Jr: The hedgehog signaling network. Am J Med Genet A 123: 5-28, 2003.

26. Dahmane N, Lee J, Robins P, Heller P and Ruiz i Altaba A: Activation of the transcription factor Gli1 and the Sonic hedgehog signalling pathway in skin tumours. Nature 389: 876-881, 1997.

27. Stein U, Eder C, Karsten U, Haensch W, Walther W and Schlag PM: GLI gene expression in bone and soft tissue sarcomas of adult patients correlates with tumor grade. Cancer Res 59: 1890-1895, 1999.

28. Van den Brink GR, Bleuming SA, Hardwick JC, Schepman BL, Offerhaus GJ, Keller JJ, Nielsen C, Gaffield W, Van Deventer SJ, Roberts DJ and Peppelenbosch MP: Indian Hedgehog is an antagonist of Wnt signaling in colonic epithelial cell differentiation. Nat Genet 36: 277-282, 2004. 